\title{
Local order around rare earth ions during the devitrification of oxyfluoride glasses
}

\author{
Maurício A. P. Silva, ${ }^{1, a)}$ Geraldine Dantelle, ${ }^{2, b)}$ Michel Mortier, ${ }^{2}$ André Monteil, ${ }^{3}$ \\ Sidney J. L. Ribeiro, ${ }^{4}$ Younès Messaddeq, ${ }^{4}$ Valérie Briois, ${ }^{5}$ and Marcel Poulain ${ }^{6}$ \\ ${ }^{1}$ Departamento de Química, Universidade Federal de Juiz de Fora, Campus Universitário S/N, Martelos, \\ Juiz de Fora, 36036-900 Minas Gerais, Brazil \\ ${ }^{2}$ Laboratoire de Chimie de la Matière Condensée de Paris, UMR 7574, ENSCP, 11 rue Curie, \\ F-75005 Paris, France \\ ${ }^{3}$ POMA, UMR 6136, Université d'Angers, 2 Bd. Lavoisier, F-49045 Angers, France \\ ${ }^{4}$ Instituto de Química, Universidade Estadual Paulista-UNESP, C.P. 355, Araraquara, \\ 14801-970 Sao Paulo, Brazil \\ ${ }^{5}$ Synchrotron SOLEIL, L'Orme des Merisiers Saint-Aubin, P.O. Box 48, 91192 Gif-sur-Yvette, France \\ ${ }^{6}$ Laboratoire des Matériaux Photoniques, Université de Rennes I, Bât. 10B, Campus de Beaulieu, 35065 \\ Rennes, France
}

(Received 1 February 2008; accepted 20 May 2008; published online 27 June 2008)

\begin{abstract}
Erbium $L_{3}$-edge extended x-ray absorption fine structure (EXAFS) measurements were performed on rare earth doped fluorosilicate and fluoroborate glasses and glass ceramics. The well known nucleating effects of erbium ions for the crystallization of cubic lead fluoride (based on x-ray diffraction measurements) and the fact that the rare earth ions are present in the crystalline phase (as indicated by $\mathrm{Er}^{3+}$ emission spectra) seem in contradiction with the present EXAFS analysis, which indicates a lack of medium range structural ordering around the $\mathrm{Er}^{3+}$ ions and suggests that the lead fluoride crystallization does not occur in the nearest neighbor distance of the rare earth ion. Molecular dynamics simulations of the devitrification process of a lead fluoride glass doped with $\mathrm{Er}^{3+}$ ions were performed, and results indicate that $\mathrm{Er}^{3+}$ ions lower the devitrification temperature of $\mathrm{PbF}_{2}$, in good agreement with the experimental results. The genuine role of $\mathrm{Er}^{3+}$ ions in the devitrification process of $\mathrm{PbF}_{2}$ has been investigated. Although $\mathrm{Er}^{3+}$ ions could indeed act as seeds for crystallization, as experiments suggest, molecular dynamics simulation results corroborate the experimental EXAFS observation that the devitrification does not occur at its nearest neighbor distance. (C) 2008 American Institute of Physics. [DOI: 10.1063/1.2943672]
\end{abstract}

\section{INTRODUCTION}

The devitrification trends of erbium-doped oxyfluoride glasses containing $\mathrm{PbF}_{2}$ have attracted the interest of scientific community due to the well known ease of control of the crystalline phase formation. ${ }^{1-5}$ This interest can be attributed to two factors. On one hand the erbium ions seem to ease the selective devitrification of $\beta-\mathrm{PbF}_{2}$ nanoparticles in the transparent glassy matrix, decreasing the amorphous to crystalline phase transition, giving rise to transparent glass ceramics. On the other hand, the rare earth ions have been found inside these nanocrystals. The optical properties of the rare earth in the crystalline matrix, with well defined emission lines (in contrast to the inhomogeneously broadened emission spectra of these ions in an amorphous matrix), are largely favored in a heavy metal fluoride crystal due to the low phonon energy found in this media. Optical amplifiers, lasers, and other optoelectronic devices are examples of technologies that explore the potential use of these systems.

Due to the decrease of the devitrification temperature induced by the presence of rare earth ions in the glass com-

\footnotetext{
a) Author to whom correspondence should be addressed. Electronic mail: mauricio.maps@gmail.com.

${ }^{b)}$ Present address: Department of Materials, University of Oxford, Parks Road, Oxford, OX1 3PH, United Kingdom.
}

position, associated with the fact that these ions seem to be concentrated inside the crystalline phase, it has been proposed that the rare earth ion acts as a nucleating agent for the $\beta-\mathrm{PbF}_{2}$ phase. Recent research ${ }^{6}$ has indicated that the tendency of formation of $\mathrm{PbF}_{2}$ : Er solid solutions can be attributed as a possible explanation for the devitrification trends and it has also been observed that other halides do not act in a similar way as fluorides. ${ }^{7}$ Nevertheless the experimental observations gathered up to now were not capable to describe unambiguously why and how some rare earth fluorides act in the devitrification dynamics. Recently, ${ }^{8}$ molecular dynamics (MD) simulations have shown that the presence of erbium ions in a $\mathrm{PbF}_{2}$ glass leads to a decrease on the devitrification temperature, in accord with experimental evidences. Moreover, an important conclusion could be drawn from these simulations: the devitrification does not start in the close vicinity of the rare earth ion, i.e., in its first or second coordination shell. Instead, the devitrification was found to begin in further distances from the rare earth. In this work we present new experimental and theoretical evidences that, in fact, the devitrification in the close vicinity of the rare earth is less complete and that considerable ordering effect is observed only at distances larger than $6 \AA$ from the rare earth ions. 
TABLE I. Composition, heat treatment, and labels used for the fluorosilicate and fluoroborate glasses and glass ceramics.

\begin{tabular}{|c|c|c|}
\hline Composition (mol) & Heat treatment & Label \\
\hline $0.4 \mathrm{SiO}_{2}-0.3 \mathrm{PbF}_{2}-0.3 \mathrm{CdF}_{2}$ & $\begin{array}{l}\text { As quenched glass } \\
440^{\circ} \mathrm{C}, 30 \mathrm{~min} \\
440^{\circ} \mathrm{C}, 60 \mathrm{~min}\end{array}$ & $\begin{array}{l}\text { S } \\
\text { ST1 } \\
\text { ST2 }\end{array}$ \\
\hline$\left(0.4 \mathrm{SiO}_{2}-0.3 \mathrm{PbF}_{2}-0.3 \mathrm{CdF}_{2}\right)_{0.99}\left(\mathrm{ErF}_{3}\right)_{0.01}$ & $\begin{array}{l}\text { As quenched glass } \\
440{ }^{\circ} \mathrm{C}, 30 \mathrm{~min} \\
440{ }^{\circ} \mathrm{C}, 60 \mathrm{~min}\end{array}$ & $\begin{array}{l}\text { SEr } \\
\text { SErT1 } \\
\text { SErT2 }\end{array}$ \\
\hline $0.4 \mathrm{~B}_{2} \mathrm{O}_{3}-0.3 \mathrm{PbF}_{2}-0.3 \mathrm{CdF}_{2}$ & $\begin{array}{l}\text { As quenched glass } \\
470{ }^{\circ} \mathrm{C}, 20 \mathrm{~min} \\
470{ }^{\circ} \mathrm{C}, 60 \mathrm{~min}\end{array}$ & $\begin{array}{l}\text { B } \\
\text { BT1 } \\
\text { BT2 }\end{array}$ \\
\hline$\left(0.4 \mathrm{~B}_{2} \mathrm{O}_{3}-0.3 \mathrm{PbF}_{2}-0.3 \mathrm{CdF}_{2}\right)_{0.99}\left(\mathrm{ErF}_{3}\right)_{0.01}$ & $\begin{array}{l}\text { As quenched glass } \\
470{ }^{\circ} \mathrm{C}, 20 \mathrm{~min} \\
470^{\circ} \mathrm{C}, 60 \mathrm{~min}\end{array}$ & $\begin{array}{l}\text { BEr } \\
\text { BErT1 } \\
\text { BErT2 }\end{array}$ \\
\hline
\end{tabular}

\section{EXPERIMENTAL}

\section{A. Glasses and glass ceramic preparation}

Pure and erbium-doped oxyfluoride glasses of molar compositions $0.4 \mathrm{SiO}_{2}-0.3 \mathrm{PbF}_{2}-0.3 \mathrm{CdF}_{2}$ ("S" sample), $\left(0.4 \mathrm{SiO}_{2}-0.3 \mathrm{PbF}_{2}-0.3 \mathrm{CdF}_{2}\right)_{0.99}\left(\mathrm{ErF}_{3}\right)_{0.01}$ ("SEr" sample), $0.4 \mathrm{~B}_{2} \mathrm{O}_{3}-0.3 \mathrm{PbF}_{2}-0.3 \mathrm{CdF}_{2}$ ("B" sample), and $\left(0.4 \mathrm{~B}_{2} \mathrm{O}_{3}\right.$ $\left.-0.3 \mathrm{PbF}_{2}-0.3 \mathrm{CdF}_{2}\right)_{0.99}\left(\mathrm{ErF}_{3}\right)_{0.01}$ ("BEr" sample) were obtained by quenching of the melts on brass molds, as reported in detail in our previous works. ${ }^{1,2}$ The vitrification process was confirmed by the absence of x-ray diffraction (XRD) Bragg peaks and the occurrence of glass-transition temperature on differential scanning calorimetry analysis. Fluorosilicate and fluoroborate glass ceramics were obtained by heat treatment at $440{ }^{\circ} \mathrm{C}$ for 30 and $60 \mathrm{~min}$ and at $470{ }^{\circ} \mathrm{C}$ for 20 and $60 \mathrm{~min}$, respectively. ${ }^{1,2}$ The heat-treated samples will hereafter be labeled with "T1" and "T2" according to the time of heat treatment, "T1" used for the samples treated for shorter time, and "T2" for those treated for longer time. Table I summarizes the compositions, heat treatments, and labels used in this work.

\section{B. EXAFS}

Extended $\mathrm{x}$-ray absorption fine structure (EXAFS) spectra were obtained in the D44 beamline of the DCI storage ring $(1.85 \mathrm{GeV}, 250 \mathrm{~mA})$ at LURE, France, at the $\operatorname{Er} L_{3}$ edge. The $\mathrm{x}$-ray absorption spectra were collected at ambient temperature in the fluorescence mode, using a monoelement fluorescence detector $\mathrm{Si}(\mathrm{Li})$. Spectra were obtained with an energy step of $2 \mathrm{eV}$ and a counting time of $5 \mathrm{~s}$. EXAFS data analysis was performed with the Michalowicz's "EXAFS POUR LE MAC" software. ${ }^{9}$ After absorption background subtraction by a linear function from the rough data, EXAFS signal was calculated using the Lengeler-Eisenberger $\operatorname{method}^{10}$ and converted to a function of wave vector $k \chi k$. The data were Fourier transformed over the $k$ range of 2.5-13 $\AA^{-1}$, using $k^{3}$ weight and a Kaiser window with $\tau$ $=2.5$ leading to a spectrum scaled in distances (angstroms). Then the peaks in the Fourier transforms corresponding to a coordination shell were filtered and transformed back to $k$-space. The resulting EXAFS-filtered signal was treated as a sum of sinusoidal wave functions using the single scattering approximation $^{11}$

$$
k \chi(k)=S_{0}^{2} \sum_{i} \frac{N_{i}}{R_{i}^{2}} e^{-2 k^{2} \sigma_{i}^{2}} e^{2 R_{i} \Gamma / k} f_{i j}(\pi, k) \sin \left[2 k R_{i}+\Phi_{i}(k)\right],
$$

where $N_{i}$ is the number of atoms in the coordination shell at average interatomic distance $R_{i}$ from the absorbing atom, $\sigma_{i}$ is the Debye-Waller factor, which takes into account the static and thermal structural disordering, $\lambda(k)$ is the photoelectron mean free path $(\lambda=k / \Gamma)$, and $f_{i j}(\pi, k)$ and $\Phi_{i}(k)$ are the amplitude and the phase functions for this coordination shell, which have been obtained experimentally from the reference $\mathrm{ErF}_{3}$ compound. Finally, a least squares fitting was implemented on the Fourier-filtered signal obtained for the glasses and glass ceramics, leading to the determination of the structural parameters $R, N$, and $\sigma$ corresponding to the respective coordination shells of the absorbing atoms. Typical accuracy in the determination of these parameters is $10 \%$ for $N, 20 \%$ for $\sigma$, and $1 \%$ for the interatomic distances. The reliability of the fit is given by the value of the residual function defined as

$$
\rho=\frac{\sum_{i} k_{i}\left[\chi_{\text {expt }}\left(k_{i}\right)-\chi_{\mathrm{th}}\left(k_{i}\right)\right]^{2}}{\sum_{i} k \chi_{\text {expt }}\left(k_{i}\right)^{2}} .
$$

\section{MD SIMULATIONS}

The classical MD simulations of the Er: $\mathrm{PbF}_{2}$ system, performed using the DL_POLY program, ${ }^{12}$ were based on a model where the interactions between two ions $i$ and $j$ were described by the Buckingham potential $U(r)$ as follows:

$$
U(r)=\frac{Z_{i} Z_{j} e^{2}}{r_{i j}}+A_{i j} \exp \left(\frac{-r_{i j}}{\rho_{i j}}\right)-\frac{C_{i j}}{r_{i j}^{6}},
$$

where $r_{i j}$ represents the distance between the ions $i$ and $j, A_{i j}$ stands for the short range repulsive coefficient, $C_{i j}$ is an interaction parameter, and $\rho_{i j}$ is an adjustable constant. The parameters concerning the interactions $\mathrm{Pb}-\mathrm{Pb}, \mathrm{Pb}-\mathrm{F}, \mathrm{Er}-\mathrm{F}$, and F-F were obtained from previous studies. ${ }^{13-16}$ For the long range electrostatic interactions, the Ewald sum technique was used.

The simulation details were described in Ref. 8. Neutrally charged face-centered cubic $\mathrm{Er}: \mathrm{PbF}_{2}$ systems of about 2590 particles, containing one and four erbium atoms (called PFE1 and PFE4, respectively) and some fluorine or lead vacancies, were melted at $3000 \mathrm{~K}$ and thermalized during a $50 \mathrm{ps}$ run. The cells were then cooled down to $300 \mathrm{~K}$ at the rate of $q=-20 \mathrm{~K} / \mathrm{ps}$ and the glasses obtained were thermalized during a $50 \mathrm{ps}$ run. Devitrification was attained by heating the glass at a rate of $q=+0.2 \mathrm{~K} / \mathrm{ps}$ from 300 to $490 \mathrm{~K}$. Total enthalpy, radial distribution functions, XRD curves, and diffusion coefficients were calculated, and simulation cell snapshots were monitored. ${ }^{8}$ 


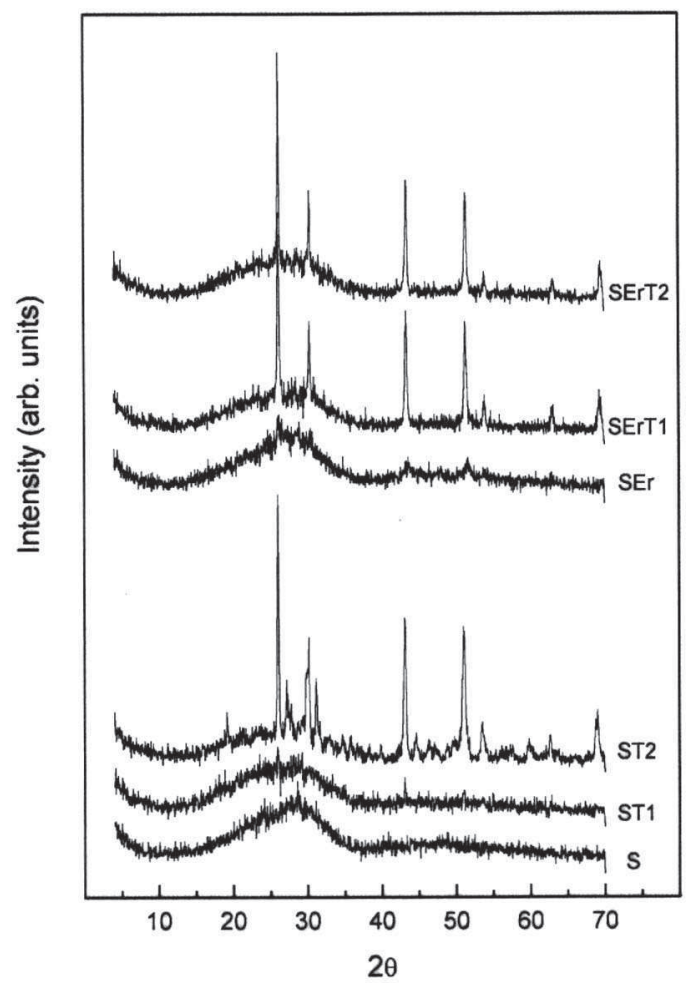

FIG. 1. XRD patterns for the fluorosilicate and fluoroborate glasses and glass ceramics (see Table I for compositions and heat treatments).

\section{RESULTS}

\section{A. Experimental}

Figure 1 shows the XRD curves for the samples of the fluorosilicate systems, the results from the fluoroborates being comparable. ${ }^{1,2}$ Cubic lead fluoride $\left[\beta-\mathrm{PbF}_{2}\right.$ (Ref. 17)] is the main crystalline form obtained from heat treatment of the samples. The presence of $\mathrm{Er}^{3+}$ ions in the glass matrix increases the crystallization trend. XRD curves indicate that the crystalline phase is observed only under a longer heat treatment for the nondoped samples, ST2, while, in the doped sample, the presence of $\beta-\mathrm{PbF}_{2}$ crystallites was detected on the SEr glass. Under the first heat treatment, SErT1, the devitrification is already well accomplished.

The presence of erbium in the crystalline phase can be proposed based on the changes of the Bragg peak positions, leading to a larger $\mathrm{PbF}_{2}$ cubic cell parameter. Additionally the near infrared $\mathrm{Er}^{3+}$ emission spectra change from the ${ }^{4} I_{13 / 2} \rightarrow{ }^{4} I_{15 / 2}$ broad transition, due to the statistical distribution of sites in the glasses, to well resolved lines characteristic of the glass ceramics.

Figure 2(a) shows the EXAFS $k \chi(k)$ spectra obtained at the Er $L_{3}$ edge of the samples. Figure 2(b) shows the corresponding Fourier transform $|F(r)|$ curves of the EXAFS signals. The peaks around $1.9 \AA$ on the $|F(r)|$ curves, uncorrected for phase shift, correspond to the first Er coordination shell composed of fluorine anions, as will be discussed below. These $|F(r)|$ curves, which express the short range ordering, do not show for all samples any structure indicating a medium range ordering.

Quantitative simulations of the inverse-Fourier trans-

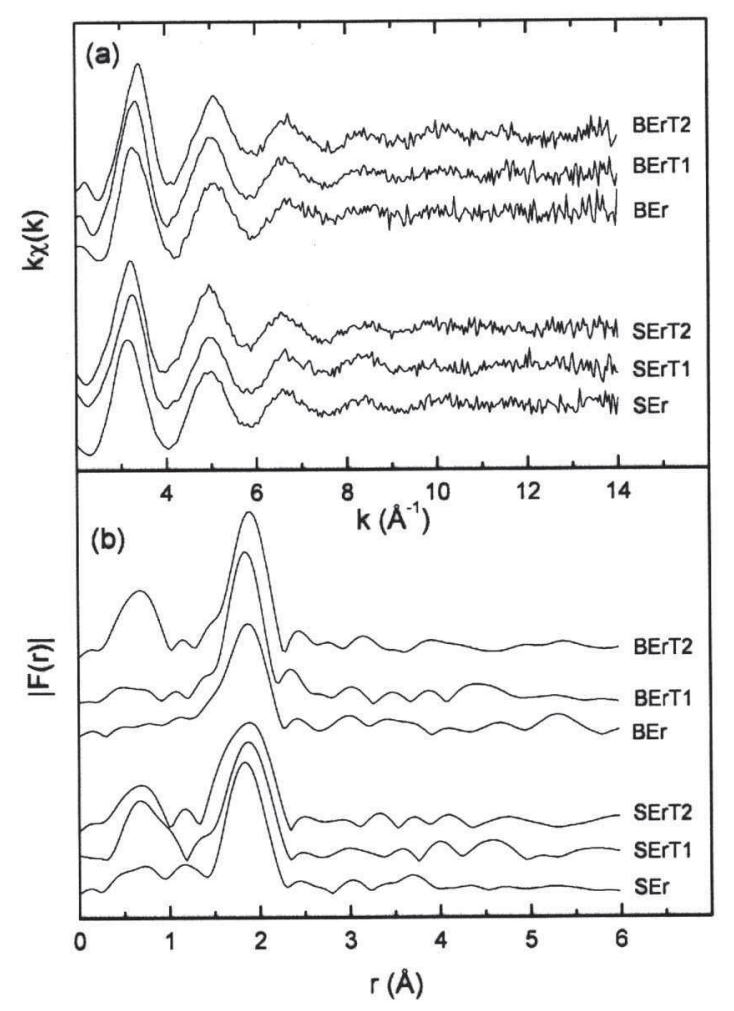

FIG. 2. (a) EXAFS $k \chi(k)$ spectra obtained at the Er $L_{3}$ edge of the samples and (b) the corresponding Fourier transform $|F(r)|$ curves of the EXAFS signals.

formed mean peak in $|F(r)|$, filtered between 1.40 and $2.35 \AA$ for the fluorosilicate samples and 1.47-2.27 $\AA$ for the fluoroborate samples, are reproduced in Fig. 3. For example, simulations for the SEr and BEr samples are shown in this figure, and the structural parameters obtained from the simulations of all samples are listed in Table II. In the glasses and glass ceramics studied, the first Er coordination shell does not change considerably from that of the reference compound $\mathrm{ErF}_{3}$, where the erbium atoms are surrounded by eight fluorine neighbors at a distance of $2.29 \AA .^{18}$

\section{B. MD simulations}

The calculation of the total enthalpy, radial distribution functions, XRD curves, diffusion coefficients, and simulation cell snapshots of the systems PFE1 and PFE4 showed that the crystallization temperature of $\mathrm{PbF}_{2}$ decreases with the increase of $\mathrm{Er}^{3+}$ ions. ${ }^{8}$ Moreover, in the simulation model, the devitrification of the PFE4 system occurs in a temperature range between 380 and $390 \mathrm{~K}$.

The simulation cell of the devitrified PFE4 system, at $390 \mathrm{~K}$, is shown in Fig. 4(a). The cell was divided in eight subcells, labeled by $(+++),(++-),(+-+)$, and so forth, depending on their position with respect to the origin of the cell, as indicated in Fig. 4(b). The diffraction intensity at each subcell of PFE4 has been calculated separately, using the Debye formula, ${ }^{8}$ and the results are reported in Fig. 4(c). Despite the fact that each subcell is at least partly crystallized, indicated by the diffraction peaks, the diffracted intensity varies from one subcell to another one. The homoge- 


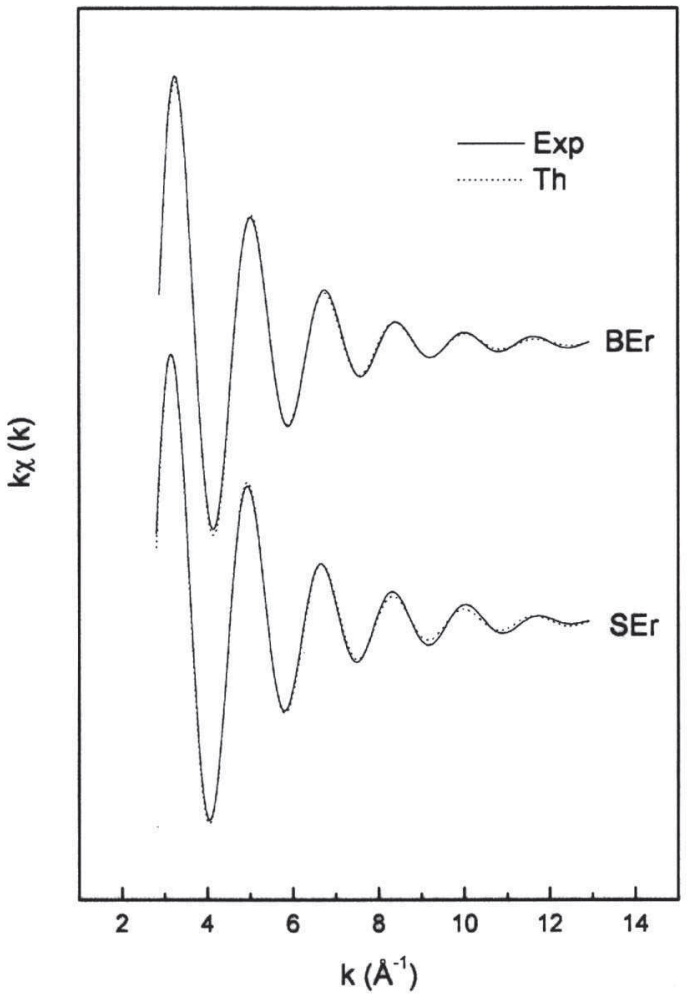

FIG. 3. Simulations of the inverse-Fourier transformed mean peak in $|F(r)|$ (first coordination shell) for the fluoroborate (BEr) and fluorosilicate (SEr) glassy samples.

neous crystallization of the system is not related to the location of $\mathrm{Er}^{3+}$ ions inside the subcells since the subcells containing $\mathrm{Er}^{3+}$ ions are those with less ordering.

Partial radial distribution functions, $g_{\alpha \beta}(r)$, were calculated for each crystallization stage, starting from the glass at $370 \mathrm{~K}$. As shown in our previous work, $\mathrm{Pb}-\mathrm{F}$ radial distribution functions confirmed the devitrification of the cubic $\beta-\mathrm{PbF}_{2}$ structure. ${ }^{8}$ Figure 5 shows the $\mathrm{Er}-\mathrm{Pb}$ radial distribution functions for the PFE4 glass at $370 \mathrm{~K}$ and for the devitrifying system at 380, 390, and $400 \mathrm{~K}$. The first peak at around $4 \AA$ indicates the first $\mathrm{Pb}$ neighbors surrounding the rare earth ions. It is clear from this figure that the first $\mathrm{Er}-\mathrm{Pb}$ shell does not change significantly from the glass to the crystallized material. On the other hand, crystallization is evidenced at longer distances by the intensification, upon devitrification, of the peaks at around 5.8 and $7.2 \AA$.

From the atomic coordinates provided by the MD simulations, it is possible to simulate the respective EXAFS spectra. MD/EXAFS spectra are obtained using the FEFF6 program, ${ }^{19}$ in which the MD coordinates are used in the
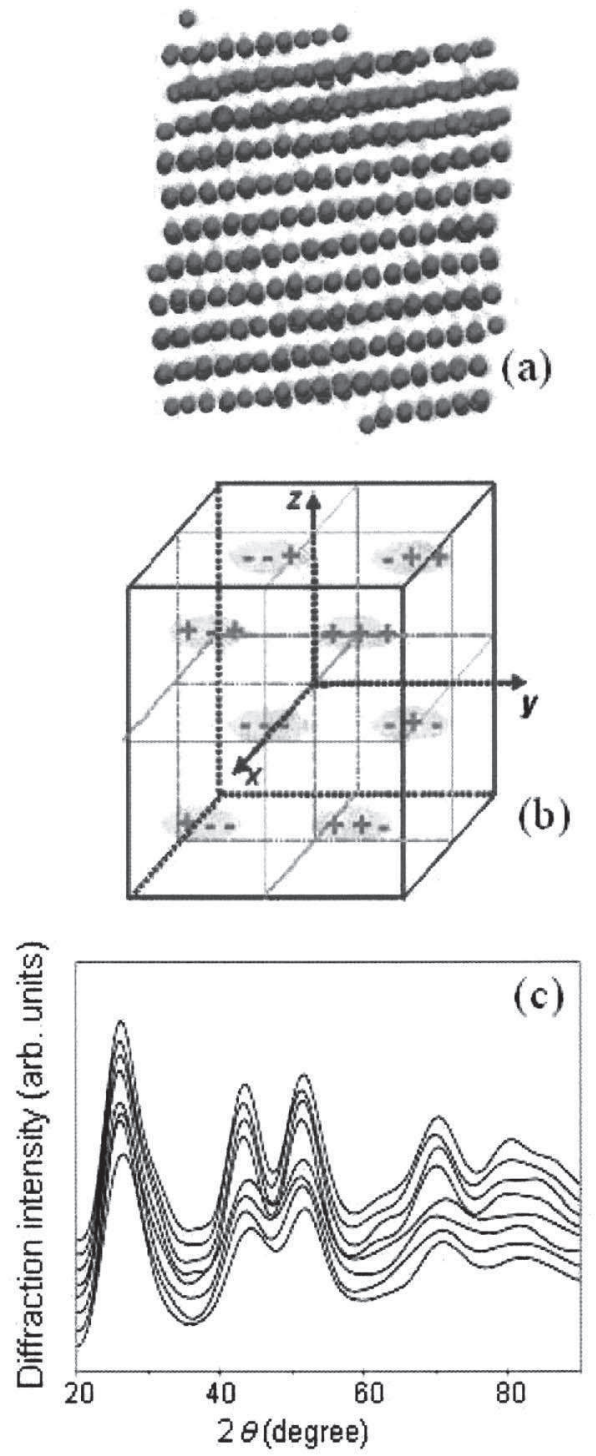

FIG. 4. (a) Snapshot ( $x-y$ axis) of PFE4 after the devitrification process at 390 K. (b) Representation of the simulation cell divided in eight subcells. (c) Diffraction intensity on each subcell at $390 \mathrm{~K}$ from high to low: (-- -), $(--+),(+--),(+-+),(++-),(-+-),(-++)$, and $(+++)$. The subcells $(-+-),(-++)$, and $(+++)$ are the ones containing at least one Er atom.

ATOMS card of the feff.inp input file. To obtain the local environment of the absorbing atom, each of the four Er atom in the MD simulation shell was chosen as the central position $\{0,0,0\}$ and the surrounding atom positions were determined by the MD configuration with respect to the central absorbing atom. The FEFF program calculates the scattering geom-

TABLE II. Structural parameters obtained from the Er $L_{3}$-edge EXAFS analysis.

\begin{tabular}{lcccccc}
\hline & SEr & SErT1 & SErT2 & BEr & BErT1 & BErT2 \\
\hline$N$ & 8.3 & 7.5 & 8.3 & 7.5 & 8.2 & 7.2 \\
$\sigma(\AA)$ & 0.109 & 0.104 & 0.111 & 0.112 & 0.106 & 0.100 \\
$R(\AA)$ & 2.25 & 2.26 & 2.26 & 2.25 & 2.27 & 2.27 \\
$\Delta E_{0}(\mathrm{eV})$ & 0.7 & 1.1 & 1.1 & -0.9 & -0.4 & 0.1 \\
$\rho$ & $1.8 \times 10^{-3}$ & $8.8 \times 10^{-4}$ & $2.4 \times 10^{-3}$ & $9.8 \times 10^{-4}$ & $5.1 \times 10^{-3}$ & $1.8 \times 10^{-3}$ \\
\hline \hline
\end{tabular}




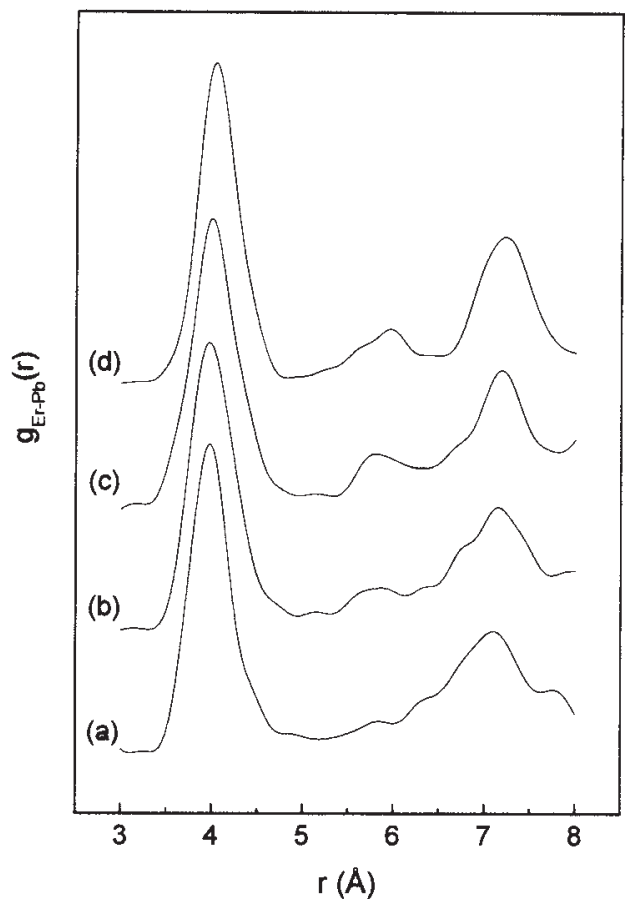

FIG. 5. Er-Pb radial distribution functions for the PFE4 glass at $370 \mathrm{~K}$ (a) and for the devitrifying system at $380 \mathrm{~K}$ (b), $390 \mathrm{~K}$ (c), and $400 \mathrm{~K}$ (d).

etries within a given radius around the absorbing atom, considering all possible single and multiple scattering paths. The total $\chi(k)$ function is then computed as a sum over all scattering paths. The $\chi(k)$ function obtained for each Er atom in the MD simulation shell, at a given temperature, was then averaged and the MD/EXAFS data, Fourier transformed using $k^{3}$ weight and a Kaiser window with $\tau=2.5$ leading to a spectrum scaled in distances (angstroms) in the same way as the experimental ones. Figures 6(a)-6(d) show the magnitude of the Fourier transformed MD/EXAFS signal obtained for the PFE4 glass at $370 \mathrm{~K}$ [Fig. 6(a)] and for the devitrifying system at $380 \mathrm{~K}$ (b), $390 \mathrm{~K}$ (c), and $400 \mathrm{~K}$ (d). As in the case of the experimental data, these $|F(r)|$ curves does not show, for all samples, any structure indicating a significant medium range ordering, such as a second coordination shell surrounding the Er atoms. In order to make clear what kind of EXAFS signal would be expected if the environment of the Er ions was crystalline, a MD simulation was performed in a fcc $\beta-\mathrm{PbF}_{2}$ structure with a lead atom position occupied by an erbium atom. The 50 ps MD simulation run was performed at $300 \mathrm{~K}$ in the NVT (constant particle number, volume, and temperature) ensemble and thermalized during another $50 \mathrm{ps}$ in the NVE (constant particle number, volume, and energy) ensemble. The Fourier transformed MD/EXAFS signal obtained for this short range crystalline Er environment is shown in Fig. 6(e). The effects of the cationic ordering in the close vicinity of the Er atom are reflected by the peak centered at $3.9 \AA$, i.e., the second $\mathrm{Er}$ coordination shell, formed by the lead atoms.

\section{DISCUSSION}

Erbium ions are often described as nucleating agents for the $\beta-\mathrm{PbF}_{2}$ crystallization (based on XRD experiments, as

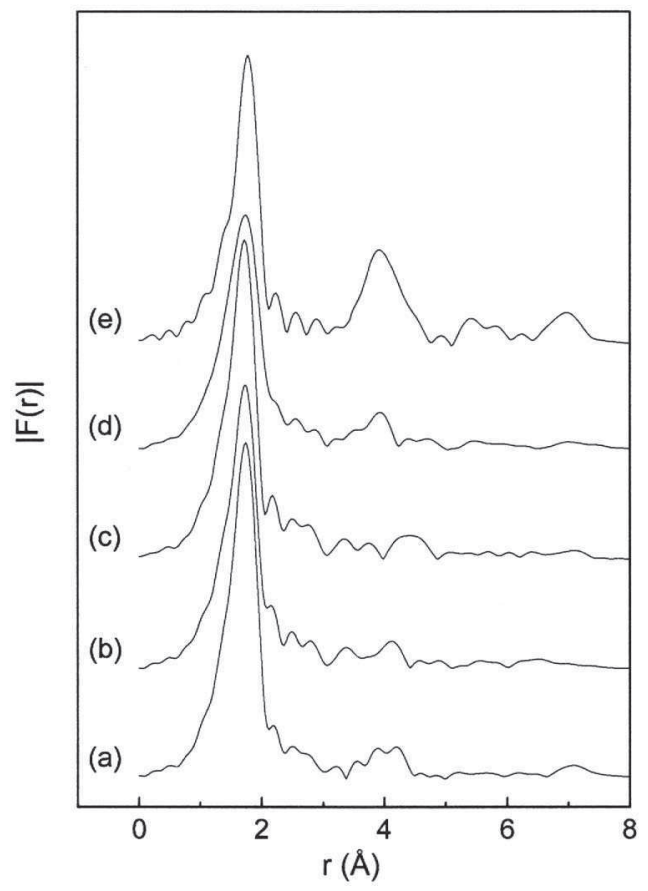

FIG. 6. The magnitude of the Fourier transformed Er $L_{3}$-edge EXAFS signal calculated from the MD configurations obtained for the PFE4 glass, averaged over the four Er atoms, at $370 \mathrm{~K}$ (a), and for the devitrifying system at $380 \mathrm{~K}$ (b), $390 \mathrm{~K}$ (c), and $400 \mathrm{~K}$ (d). The Fourier transformed EXAFS signal in (e) is related to a single Er atom occupying a lead crystallographic position in the fcc crystalline $\beta-\mathrm{PbF}_{2}$ matrix.

shown above), explaining their presence in the crystalline phase (as, for instance, indicated by $\mathrm{Er}^{3+}$ emission spectra experiments). The description of $\mathrm{Er}^{3+}$ as a nucleating agent seems to imply that the nucleation of $\mathrm{PbF}_{2}$ starts around $\mathrm{Er}^{3+}$ ions. However, EXAFS analysis indicates that except from the first coordination shell of the Er atoms composed by fluorine ions, no structural ordering is observed as a second coordination shell.

In fact, if the devitrification occurred in a very close distance from the rare earth ion, i.e., the Er ions acting as a nucleating agent for the heterogeneous crystallization, retrodiffusion of $\mathrm{Pb}$ scatterers would have been observed on the EXAFS spectra, as a second peak on the Fourier transformed signal [see Fig. 6(e)]. Figure 2 does not show this feature, which indicates that the lead fluoride crystallization does not occur in the close vicinity of the rare earth ion.

The simulation results ${ }^{8}$ indicate that while the devitrification temperature $T_{c}$ depends on the number of $\mathrm{Er}^{3+}$ incorporated in the glasses (higher $\mathrm{Er}^{3+}$ content, lower $T_{c}$ ), the devitrification seems to be less efficient around the doping ions. In fact, the evolution of the $g_{\mathrm{ErPb}}(r)$ curves during devitrification, obtained by MD simulations and depicted in Fig. 5, shows clearly that the short range order around the doping ions $(R<5 \AA)$ does not reveal any structural change upon crystallization. On the other hand, the main structural features concerning crystallization around the Er ions are located at distances larger than $6 \AA$, the main peak appearing at distances larger than $7 \AA$. Due to the finite mean free path of the photoelectron that limits the EXAFS technique to local order configurations typically shorter than $6 \AA$, the distances 
from the rare earth ions at which the crystallization occurs do not allow the detection of this structural evolution. Our MD simulations were able to reproduce this behavior with accuracy, as shown in Fig. 5.

A close report between MD simulations and EXAFS technique could be drawn by the use of the MD configurations to simulate EXAFS $\chi(k)$ functions. Despite the fact that some ordering is indeed observed around $4 \AA$ in the MD/ EXAFS data of Figs. 6(a)-6(d), related to the lead atoms around the central Er atoms, the agreement with the experimental result, as shown in Fig. 2(b), is noteworthy.

As it is well known, the damping effects of the thermal motions on the EXAFS equation [represented, in part, by the Debye-Waller factor $\sigma_{i}$ in Eq. (1)] limit the backscattering effects perceived by the absorbing atoms, which make the detection of the second coordination shell in the Fourier transformed EXAFS $\chi(k)$ signal difficult. However, it must be kept in mind that the MD configuration used in the MD/ EXAFS calculations represents an instantaneous, static picture of the system at that temperature, in comparison to the dynamical, ambient temperature system, from which the experimental data were extracted. In other words, the MD/ EXAFS spectra of Figs. 6(a)-6(e), obtained via MD configurations, are not affected by thermal vibrations. Our MD/ EXAFS results show that the thermal effect is not the factor that limits the experimental observation of structural ordering around the Er atoms only to the first fluorine coordination shell. In this case, the intensity degree of the peak at around $4 \AA$, representing the second coordination shell in the Fourier transformed EXAFS signals [Fig. 2, 5, and 6(e)], is dependent mainly on the statistical disorder of the scatterer $\mathrm{Pb}$ atoms. Actually, the peak observed at around $4 \AA$ in the Fourier transformed EXAFS signal of Fig. 6(e) corroborates our assumptions that, had the devitrification started in the close vicinity of the Er atom and the cationic crystallographic positions statistically occupied around the rare earth, the presence of a second coordination shell formed by the lead atoms would be identified by the EXAFS analysis. Indeed, this MD/EXAFS data treatment reinforces the conclusions drawn by the experimental EXAFS observations and corroborates the lack of structural ordering in the close vicinity of Er atoms computed by the MD simulations.

Several factors are yet unclear and must be evaluated to draw a precise conclusion about the genuine role of $\mathrm{Er}^{3+}$ ions in the devitrification process of $\mathrm{PbF}_{2}$. One of these factors, which is yet to be tested experimentally and theoretically, is the possibility of an amorphous-amorphous phase separation induced by the rare earth ion, creating nanosized $\mathrm{PbF}_{2}$-rich regions around the rare earth. In this case, the lack of the structural evolution during crystallization could be attributed to the fact that the devitrification itself occurs not in the immediate neighborhood of the rare earth ion but in the interface between the two amorphous phases, leaving the rare earth trapped inside the crystal, but with a considerable disorder in its close vicinity due to the direction of the crystalline growth.

\section{CONCLUSION}

In this work, MD simulations were performed on the devitrification properties of Er-doped lead fluoride glass, and the results were unambiguously confirmed by $\operatorname{Er} L_{3}$-edge EXAFS measurements. The fact that the crystallization of $\beta-\mathrm{PbF}_{2}$ does not occur at the immediate neighborhood of the rare earth ion is in contradiction with the widely spread theory that these trivalent ions act as a nucleating agent for this crystalline phase. Experimental and theoretical results indicate that the erbium ions indeed act on the crystallization behavior of fluoride and oxyfluoride glasses, but simply inducing a decrease of the crystallization temperature that should reflect the tendency to form a $\mathrm{PbF}_{2}-\mathrm{ErF}_{3}$ solid solution. The nucleation role of these ions on the heterogeneous crystallization of the glasses was not confirmed by the EXAFS measurements and by the MD simulations presented due to the lack of cationic structural ordering around the rare earth. The use of MD configurations to simulate EXAFS spectra is well suited for examining the convergence of both theoretical and experimental techniques and was used in this work to confirm that the lack of structural short-range ordering around the Er atoms, observed by MD and EXAFS, is not an issue of thermal damping effects.

\section{ACKNOWLEDGMENTS}

The financial support of FAPEMIG, FAPESP, CNPq, and CAPES (Brazilian agencies) is gratefully acknowledged.

${ }^{1}$ M. A. P. Silva, V. Briois, M. Poulain, Y. Messaddeq, and S. J. L. Ribeiro, J. Phys. Chem. Solids 64, 95 (2003).

${ }^{2}$ M. A. P. Silva, S. J. L. Ribeiro, Y. Messaddeq, V. Briois, and M. Poulain, J. Braz. Chem. Soc. 13, 200 (2002).

${ }^{3}$ L. A. Bueno, P. Melnikov, Y. Messaddeq, and S. J. L. Ribeiro, J. NonCryst. Solids 247, 87 (1999).

${ }^{4}$ G. Dantelle, M. Mortier, G. Patriarche, and D. Vivien, J. Solid State Chem. 179, 1995 (2006).

${ }^{5}$ M. Mortier and F. Auzel, J. Non-Cryst. Solids 256-257, 361 (1999).

${ }^{6}$ I. I. Buchinskaya and P. P. Fedorov, Russ. Chem. Rev. 73, 371 (2004).

${ }^{7}$ M. Mortier, C. Chateau, M. Génotelle, and N. Gardant, J. Non-Cryst. Solids 326-327, 287 (2003).

${ }^{8}$ G. Dantelle, M. Mortier, A. Monteil, S. Chaussedent, and M. A. P. Silva, J. Chem. Phys. 127, 094509 (2007).

${ }^{9}$ A. Michalowicz, EXAFS pour le Mac (Societé Française de Chimie, Paris, 1991), p. 102.

${ }^{10}$ B. Lengeler and P. Eisenberger, Phys. Rev. B 21, 4507 (1980).

${ }^{11}$ B. K. Teo, EXAFS: Basic Principles and Data Analysis, Inorganic Chemistry Concepts, Vol. 9 (Springer, Berlin, 1986).

${ }^{12}$ W. Smith and T. R. Forester, DL_POLY_2 User Manual, (The Council for the Central Laboratory of the Research Councils, Daresbury Laboratory at Daresbury, Nr. Warrington, 2003).

${ }^{13}$ A. B. Walker, M. Dixon, and M. J. Gillan, J. Phys. C 15, 4061 (1982).

${ }^{14}$ M. E. G. Valerio, R. A. Jackson, and J. F. Lima, J. Phys.: Condens. Matter 12, 7727 (2000).

${ }^{15}$ M. A. P. Silva, A. Monteil, Y. Messaddeq, and S. J. L. Ribeiro, J. Chem. Phys. 117, 5366 (2002).

${ }^{16}$ M. A. P. Silva, J. P. Rino, A. Monteil, S. J. L. Ribeiro, and Y. Messaddeq, J. Chem. Phys. 121, 7413 (2004).

${ }^{17}$ JCPDS Card No. 6-0251.

${ }^{18}$ R. W. Wyckoff, Crystal Structures, 2nd ed. (Krieger, Malabar, Florida, 1982), Vol. 1, p. 241.

${ }^{19}$ S. I. Zabinsky, J. J. Rehr, A. Ankudinov, R. C. Albers, and M. J. Eller, Phys. Rev. B 52, 2995 (1995). 